\title{
Spectroscopic Study of Cytosine Methylation Effect on Thermodynamics of DNA Duplex Containing CpG Motif
}

\author{
Václav Římal, Ondřej Socha, Josef Štěpánek, and Helena Štěpánková \\ Faculty of Mathematics and Physics, Charles University in Prague, Ke Karlovu 3, 12116 Prague 2, Czech Republic \\ Correspondence should be addressed to Josef Štěpánek; stepjos@karlov.mff.cuni.cz
}

Received 6 February 2015; Revised 10 August 2015; Accepted 11 August 2015

Academic Editor: Eugen Culea

Copyright (c) 2015 Václav Rímal et al. This is an open access article distributed under the Creative Commons Attribution License, which permits unrestricted use, distribution, and reproduction in any medium, provided the original work is properly cited.

Effect of cytosine methylation on DNA duplexes was studied by using a model system of three self-complementary DNA octamers containing central CpG motif surrounded by a couple of AT base pairs, CAACGTTG, CATCGATG, and CTTCGAAG, and their analogues with the central cytosine methylated at $\mathrm{C} 5$ position. Temperature dependences of ${ }^{1} \mathrm{H}$ NMR, UV absorption, and Raman scattering spectra measured for aqueous solutions at concentrations of different orders of magnitude were subjected to a joint analysis that allowed an accurate determination of the enthalpy and entropy of duplex formation. It was revealed that the changes of the enthalpy and entropy contributions are strongly dependent on the base composition in the vicinity of the CpG motif.

\section{Introduction}

Methylation of cytosine at carbon position C5 is a highly abundant epigenetic chemical modification of DNA in mammals, occurring mainly in $\mathrm{CpG}$ dinucleotide sequences [1]. Cytosine methylation generally triggers or prevents many protein-DNA interactions involved in diverse biochemical processes, including mediation of gene repression $[2,3]$. On the other hand, deoxynucleotide sequence containing nonmethylated cytosine is recognized by toll-like receptor 9 , which starts an immune response $[4,5]$. The use of the CpG containing oligonucleotides as immunostimulatory agents in vaccines was thus proposed and tested [6,7].

Despite the large number of known cases where DNAprotein interaction depends on the methylation state of the target DNA, the mechanism of the recognition is not fully understood. The initial recognition feature is naturally sought in the effect of the cytosine methylation on geometry, dynamics, and/or thermal stability of DNA duplex. It is generally accepted that the cytosine methylation stabilizes the duplex. This was clearly demonstrated by recent studies of temperature induced melting of larger DNA segments (from several tens to hundreds of nucleotides) monitored by means of intercalating fluorescence probe [8-10]. Methylation of some fraction of cytosines (without any specification of their flanking nucleosides) caused distinct several centigrades increases of the annealing temperature.

On the other hand, more detailed studies performed on shorter deoxyoligonucleotides containing the CpG motif revealed only weak structural and stability changes. In particular, recent thorough studies on Drew-Dickerson dodecamer by means of X-ray diffraction completed with NMR or CD and UV absorption measurements [11, 12] have shown that the basic spatial structure of the double helix is only very weakly affected by the cytosine methylation. The duplex retained the original B-type conformation. The thermal stability was also found as indistinguishable except a single case of increase in the melting temperature about $1.3^{\circ} \mathrm{C}$ [11]. The only remarkable effect of the cytosine methylation on the Drew-Dickerson dodecamer reported so far was a reduced amplitude of motions of the sugar-phosphate backbone indicated by solid-state NMR [13]. Similar results were also obtained on a few other CpG motifs containing oligonucleotide sequences: no significant structural modification but indication of the dynamics reduction in [14] and only weak increase of the thermal stability after the methylation (melting temperature increase of $1.4^{\circ} \mathrm{C}$ ) in [15].

Certain changes in the $\mathrm{CpG}$ local geometry by the cytosine methylation were found in the nineties by NMR studies performed on oligonucleotides where the $\mathrm{CpG}$ motif was surrounded by two AT nucleotide pairs from both sides. 
These works revealed that in this case the $\mathrm{CpG}$ site conformation was not sufficiently locked in a standard B-form geometry even without the cytosine methylation [16-18]. The conformational variability of the CpG dinucleotide segment in respect of its nucleotide surroundings was recently confirmed by statistical analysis of experimental structural data and of molecular dynamics trajectories made by the $\mathrm{ABC}$ group of laboratories $[19,20]$. The $\mathrm{CpG}$ dinucleotide exhibited apparent bimodal distribution of the twist. Unfortunately the authors did not distinguish in their analysis between different purines and pyrimidines and their results cannot thus say anything about the possible significant role of AT pairs.

Cytosine methylation was reported to cause local displacements that were detectable but different for each particular sequence containing AT..AT [21], TT..AA [21], or AA..TT [22] self-complementary flanking nucleotides (two dots are used instead of CG for a better clarity according to [20]). Also the thermal stability monitored by temperature dependence of NMR spectra differed: while the methylation caused $3.2^{\circ} \mathrm{C}$ increase of the melting temperature for AT..AT, an opposite effect (decrease of $1.2^{\circ} \mathrm{C}$ ) was observed for TT..AA [21].

Undoubtedly for any biomolecular interaction, including the cytosine methylation effect on DNA duplex, knowledge of the thermodynamic characteristics is significantly more useful than sole information on the thermal stability [23]. Even processes leading to small changes in Gibbs free energy (and consequently in the melting temperature) can involve a large redistribution of enthalpy and entropy contributions. Obtaining reliable values of the enthalpy and entropy changes attributed to the DNA duplex formation is unfortunately hindered by a large mutual correlation of their values. It is known, for example, that it is virtually impossible to obtain reasonable estimates of enthalpy and entropy components from analysis of a single melting curve that tracks temperature induced variation in the ratio of duplexes and single strands. On the opposite, to obtain enthalpy and entropy data as the realistic separate characteristics of the interaction under study, it is essential to collect and analyze data obtained in a wide range of determining parameters (oligonucleotide concentration in our case) and to pay high attention to minimization of experimental errors ([24] and references therein).

Aim of the presented work is to obtain the thermodynamic characteristics of cytosine methylation in the $\mathrm{CpG}$ motif in dependence on the composition of the flanking AT base pairs. We chose self-complementary DNA sequences with a central $\mathrm{CpG}$ dinucleotide surrounded by a couple of variously arranged AT pairs from both sides. In order to enlarge the concentration window we combined results from three spectroscopic techniques applicable in different concentration ranges, namely, UV absorption, NMR, and Raman scattering. Special attention was paid to a precise determination of concentrations and temperatures and to a rigorous determination of melting temperatures from experimental data.

\section{Experiment}

2.1. Samples. The study was carried out by using a model set of three deoxynucleotide octamers with self-complementary sequences and central CpG motif, namely, CAACGTTG (OctAA), CATCGATG (OctAT), CTTCGAAG (OctTT), and their analogues with the central cytosine methylated at C5 position, CAAm ${ }^{5}$ CGTTG (mOctAA), CATm ${ }^{5}$ CGATG (mOctAT), and CTTm ${ }^{5}$ CGAAG (mOctTT). The abbreviations shown in the parentheses are used throughout the text. Purified octamers were purchased from the Proteomics Group of CEITEC, Brno, Czech Republic.

Samples for all measurements were prepared as solutions in sodium phosphate buffer ( $\mathrm{pH} 7.0,25 \mathrm{mM}$ phosphate) with sodium chloride added to reach the total concentration $200 \mathrm{mM}$ of sodium cations. In the case of NMR measurements, the buffer was prepared in $\mathrm{H}_{2} \mathrm{O}: \mathrm{D}_{2} \mathrm{O}$ mixture of $9: 1$ ratio and a small amount of DSS (sodium 4,4-dimethyl-4silapentane-1-sulfonate) was added as an internal chemical shift standard. For UV and Raman spectroscopy, the octamer solutions were annealed at $80^{\circ} \mathrm{C}$ for $10 \mathrm{~min}$ and slowly cooled down to room temperature prior to filling the cuvettes.

2.2. UV Absorption. Absorption spectra in the wavelength range from $230 \mathrm{~nm}$ to $340 \mathrm{~nm}$ were acquired on Lambda 12 (PerkinElmer) double-beam spectrophotometer in standard $10 \mathrm{~mm}$ cuvettes. A drop of mineral oil was placed on the solution level to prevent evaporation at high temperatures. The sample chamber of the spectrometer was continuously flushed with dry air to avoid water condensation on the outer surface of the cuvette at low temperatures. UV absorption spectra were measured at temperatures increasing from $0^{\circ} \mathrm{C}$ to $74^{\circ} \mathrm{C}$ in $3^{\circ} \mathrm{C}$ steps. After reaching the target temperature, a 10 min waiting before the spectral measurement was used to equilibrate the temperature.

2.3. Raman Scattering. Spectra were excited by the $532.2 \mathrm{~nm}$ line of a Nd:YAG laser with frequency doubling (Verdi V2, Coherent) yielding approximately $0.5 \mathrm{~W}$ power at the sample. Scattered light collected in right-angle geometry was analyzed by a single grating spectrograph (Spex 270M, JobinYvon, 1800 grooves $/ \mathrm{mm}$ grating) with a liquid nitrogen cooled CCD detector. An edge filter in front of the spectrograph was used to suppress the elastic scattering. Raman spectra were recorded in the region of Stokes shifts 500$1824 \mathrm{~cm}^{-1}$ with $1 \mathrm{~cm}^{-1}$ resolution. Total acquisition time for one spectrum was $1000 \mathrm{~s}$. Sample, octamer solution in a $12 \mu \mathrm{L}$ cylindrical quartz microcuvette, was placed into a thermostabilized chamber. Raman spectra were measured at temperatures from $2^{\circ} \mathrm{C}$ to $80^{\circ} \mathrm{C}$ in $2^{\circ} \mathrm{C}$ steps; $10 \mathrm{~min}$ waiting time was used after reaching the target temperature for the temperature equilibration. After each measurement, spectrum of a neon lamp was recorded for precise spectral calibration. The background correction of Raman spectra was performed by subtracting optimal fifth-degree polynomial function and properly scaled solvent Raman spectra (buffer and pure water) and the spectrum of the microcell quartz.

2.4. NMR. The NMR experiments were performed on a Bruker Avance 500 spectrometer working at ${ }^{1} \mathrm{H}$ resonance frequency of $500.13 \mathrm{MHz}$. One-dimensional ${ }^{1} \mathrm{H}$ NMR spectra were acquired in the temperatures decreasing from $81^{\circ} \mathrm{C}$ 
to $0^{\circ} \mathrm{C}$ with steps of $2^{\circ} \mathrm{C}$ using double spin-echo pulse sequence with excitation sculpting by $4 \mathrm{~ms}$ selective $\pi$-pulses on water resonance and field gradient pulses to suppress solvent signal. 256 or 1024 scans (depending on the line broadening caused by chemical exchange) were collected at each temperature with acquisition times at least $1.4 \mathrm{~s}$ and recycle delays $1 \mathrm{~s} .{ }^{1} \mathrm{H}$ chemical shifts were referenced to the internal standard $\left(\delta_{\mathrm{DSS}}=0 \mathrm{ppm}\right)$. Phase correction, linear baseline correction, and no apodization window were applied to all $1 \mathrm{D}{ }^{1} \mathrm{H}$ spectra. Signals were assigned using intra- and internucleotide crosspeaks in ${ }^{1} \mathrm{H}-{ }^{1} \mathrm{H}$ NOESY spectra measured between $9^{\circ} \mathrm{C}$ and $11^{\circ} \mathrm{C}$ following standard procedures [25-27]. ${ }^{31} \mathrm{P}$ NMR spectra were acquired at $25^{\circ} \mathrm{C}$ with recycle delay $50 \mathrm{~s}$ and no decoupling. Before integration, exponential line broadening of $0.5 \mathrm{~Hz}$ was applied, phase was carefully manually optimized, and baseline automatically was corrected by a second-order polynomial.

2.5. Determination of Concentrations. In NMR spectroscopy, we determined the sample concentrations for all samples directly in the NMR tubes. ${ }^{31} \mathrm{P}$ NMR spectra were recorded using sufficiently long repetition (50 s) and acquisition ( $2 \mathrm{~s}$ ) times without decoupling to obtain accurate intensities of NMR lines. The total integral intensity of the seven phosphate peaks from the octamer backbone was compared with the inorganic phosphate signal of the buffer, used as intensity reference. For UV spectroscopy, we employed the extinction coefficients for $260 \mathrm{~nm}$ predicted by the nearest neighbor method [28] for nonmethylated octamers. We used both kinds of extinction coefficients, the ones of duplexes for $\mathrm{UV}$ absorption at low temperatures $\left(3^{\circ} \mathrm{C}-4^{\circ} \mathrm{C}\right)$ and those of single strands for $\mathrm{UV}$ absorption at a temperature sufficiently above the melting $\left(64^{\circ} \mathrm{C}\right)$, and the resulting concentration value was taken as the average. Because of lack of literature data concerning the effect of the cytosine methylation on UV absorption, we determined the extinction coefficient for mOctAA (and for OctAA as a reference) by interconnection of NMR and UV absorption capabilities. Based on the concentrations evaluated from ${ }^{31} \mathrm{P}$ NMR, this approach enabled independent determination of extinction coefficients after measurement of UV absorption on 50-fold diluted solutions. We found out that (i) the determined extinction coefficient for OctAA sample differed from the literature value [28] of less than $2 \%$, which corresponded to the precision of our method we had estimated and (ii) the methylation practically did not change the extinction coefficient value (deviation less than 2\%). Therefore, we used the same extinction coefficient values for the methylated octamers as for their nonmethylated analogues. The concentrations of samples used in Raman scattering were determined by UV absorbance after high dilution. We estimate the accuracy of the determined concentrations as $2 \%$ for the samples used in UV absorption and NMR measurements and 5\% for those used in Raman experiments.

2.6. Determination of Temperatures. During measurements of UV absorption the temperature was measured by a calibrated thermocouple placed directly in the solution. We estimated that errors of the temperature determination did not exceed $0.5^{\circ} \mathrm{C}$. In case of Raman measurement the microcuvette was placed in a thermally isolated chamber with quartz windows. The temperature was measured by a platinum thermometer in the inner thermostated metallic block surrounding the microcuvette. Tests with a thermocouple placed inside the microcuvette confirmed that the difference between the solution inside the microcuvette and the metallic block was less than $0.2^{\circ} \mathrm{C}$. We estimated the total error for Raman experiment as less than $0.7^{\circ} \mathrm{C}$. In NMR spectrometer, the temperature was indicated by a thermocouple in the probe and recalculated to the real sample temperature using calibration based on ${ }^{1} \mathrm{H}$ NMR spectra of methanol $\left(0^{\circ} \mathrm{C}-\right.$ $\left.55^{\circ} \mathrm{C}\right)$ and ethyleneglycol $\left(40^{\circ} \mathrm{C}-85^{\circ} \mathrm{C}\right)$. The calibration data were measured at the same gas flow and the temperature unit settings as spectra of octamer solutions. By this way, precision of $0.5^{\circ} \mathrm{C}$ was achieved [29].

\section{Results and Discussion}

The three series of temperature dependent spectra obtained for each octamer by means of the three spectroscopic techniques were first analyzed independently to obtain the melting temperatures for particular concentrations. The determination was based on a spectral parameter, $p$, directly tied to the fraction of melted duplexes, $\theta$. For self-complementary strands of total concentration $c$, this obeys the relation $[30,31]$

$$
\theta=\frac{\sqrt{1+8 K_{a} c}-1}{4 K_{a} c},
$$

where $K_{a}$ is the association equilibrium constant which depends on temperature according to the van't Hoff equation

$$
K_{a}=\frac{1}{c_{\text {ref }}} \exp \left(\frac{-\Delta H+\Delta S T}{R T}\right),
$$

where $\Delta H$ and $\Delta S$ are the enthalpy and entropy of the duplex association, $R$ is the molar gas constant, and $c_{\text {ref }}$ is a concentration reference, which is conventionally chosen as a unity in molar concentration scale; that is, $c_{\text {ref }}=1 \mathrm{M}$.

The melting curve, that is, the temperature dependence of $p$, which exhibits very often linear instead of constant asymptotes, can be expressed as

$$
p=\left(a_{d}+b_{d} T\right)(1-\theta)+\left(a_{s}+b_{s} T\right) \theta .
$$

The spectral parameters derived from our experimental data were subjected to least square fits according to (3) by Asymexfit toolbox (version 2.3) [32]. The fits yielded, besides the values of the free parameters $a_{d}, b_{d}, a_{s}$, and $b_{s}$, the thermodynamic quantities $\Delta H$ and $\Delta S$. Despite very good agreements between the experimental and fitted melting curves, $\Delta H$ and $\Delta S$ values obtained by this way are inaccurate due to their strong correlation when minimizing the sum of the squared deviations. They were used only as auxiliary parameters for determination of the melting temperature (at which half of the duplexes are separated; that is, $\theta=0.5$ ) according to

$$
T_{m}=\frac{\Delta H}{\Delta S+R \ln \left(c / c_{\mathrm{ref}}\right)} .
$$




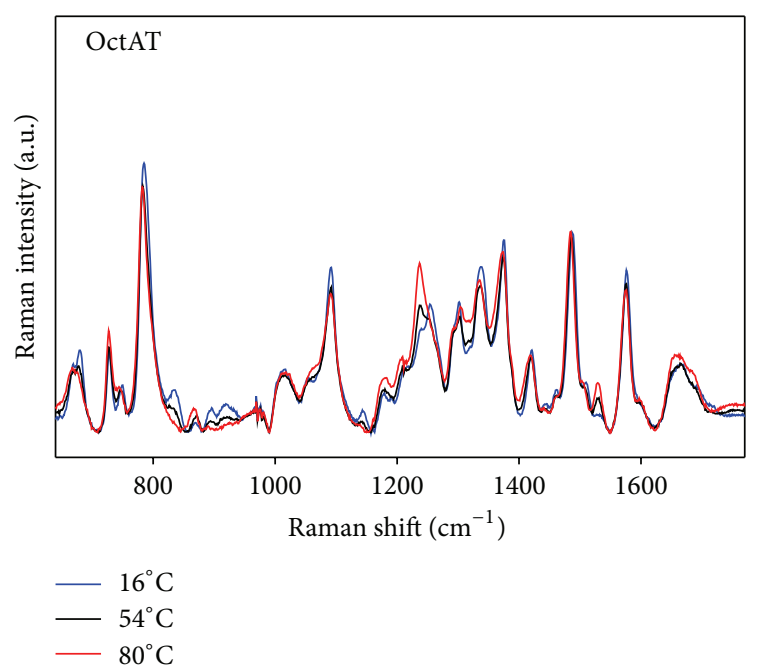

FIgURE 1: Typical background-corrected Raman spectra of selfcomplementary DNA octamer (data for OctAT) at temperatures when duplexes are formed (in blue), in the melting region (in black), and when only separated strands are present (in red).

The errors of the melting temperatures were estimated from the covariance matrices between $\Delta H$ and $\Delta S$ obtained from the fits; the errors are quite small because of the large mutual compensation of $\Delta H$ and $\Delta S$ deviations.

We neglected a possible temperature dependence of the enthalpy and entropy in our fits; that is, we assumed no change of the heat capacity upon melting. This simplification should have only a minor effect since the melting temperatures of our samples differ only by a few centigrades. Any compensation by employing published data about the heat capacity change would solely shift our results in concert while the differences between them, which are of the key importance, would remain unperturbed.

Temperature induced differences in UV absorption spectra consist, as is well known, of hypochromic change of the long-wavelength absorption band (spectra not shown). The absorbance at $260 \mathrm{~nm}$, a standard parameter, was subjected to the fit. No weights were used, assuming that the experimental error was the same for all temperatures.

In the case of Raman spectra the temperature induced duplex melting causes remarkable changes of the spectrum, mainly intensity changes or diminishing of certain lines (see Figure 1). To extract the main spectral signatures connected with the melting, each set of Raman spectra was subjected to a singular value decomposition (SVD) [33]. This method provides multivariate analysis of a set of $N$ experimental spectra $Y_{i}(\nu), i=1, \ldots, N$ ( $\nu$ represents the spectral variable), via their transformation into another set of $N$ orthonormal (mathematically independent) spectral components $U_{j}(\nu)$, $j=1, \ldots, N$, as

$$
Y_{i}(\nu)=\sum_{j=1}^{M} W_{j} V_{i j} U_{j}(\nu) .
$$

$V_{i j}$ element of the unitary matrix $\mathbf{V}$ indicates relative contribution of $j$ th spectral component to $i$ th experimental spectrum (score) and the singular value $W_{j}$ the statistical weight of $j$ th spectral component. The spectral components are ordered in a descending sequence of the corresponding singular values and the spectral set can usually be well approximated by taking into account only a few terms at the right side of (5), that is, for $M \ll N$.

Figure 2 shows typical SVD results for the temperature dependence of octamer Raman spectra. The singular numbers reveal that the spectral set can be satisfactorily expressed as a superposition of three spectral components. The first component represents an average Raman spectrum and is practically insensitive to the temperature. The spectral changes connected with the duplex melting are dominantly represented by the second spectral component and the corresponding scores $\left(V_{2}\right)$ show a typical melting curve. The third spectral component represents only weak correction of Raman spectra for unequal spectral changes caused by the temperature increase in the region below the melting and those in the melting region. The melting temperature was thus determined by a least square fit of $V_{2}$ temperature dependence according to (3).

The temperature induced changes in ${ }^{1} \mathrm{H}$ NMR spectra concern both the chemical shifts of particular hydrogen lines and their lineshapes (see Figure 3). $\theta$ values were determined separately for individual nonexchangeable nucleobase protons, that is, purine $\mathrm{H} 8$, pyrimidine $\mathrm{H} 6$, adenine $\mathrm{H} 2$, and thymine and 5-methylcytosine methyl group $\mathrm{H} 7$, by the spectral shape fitting performed on spectral regions covering several close-lying resonances. Below and above duplex melting, Lorentzian shapes were used for all peaks. During the melting (for temperatures between approximately $30^{\circ} \mathrm{C}$ and $60^{\circ} \mathrm{C}$ ), where the chemical exchange strongly influences the spectra, the asymmetrical two-site chemical exchange model was chosen to express the lineshape functions [32]. Chemical shifts of pure duplex and pure single strands were linearly extrapolated from the temperatures outside the melting region. The corresponding linewidths were taken as minima of the values obtained for the duplex region and as means of the values obtained for single strands. The fits of the free parameters, which included the intensity, fraction of melted duplexes $(\theta)$, and the dissociation rate independently for each resonance considered, were performed in the MATLAB environment using the Asymexfit toolbox (version 2.3) as described in [32]. Errors of the fitted parameters were estimated by repeating fits with modified linewidths and chemical shifts within their prediction intervals and with an artificial noise added to simulated datasets corresponding to the best fits [34].

The melting temperatures were determined by weighted least square fits of obtained $\theta$ values to (1) (the errors of $\theta$ used as weights) followed by calculations according to (3). This procedure was applied to all analyzed resonances and the resulting global $T_{m}$ value was assessed as the mean of particular $T_{m}$ 's weighted according to their errors.

Table 1 shows the melting temperatures determined for the six octamers by the three spectroscopic techniques. The given errors were derived as combinations of the errors coming from the fits and the estimated experimental errors of the temperature measurements. Assuming independent 

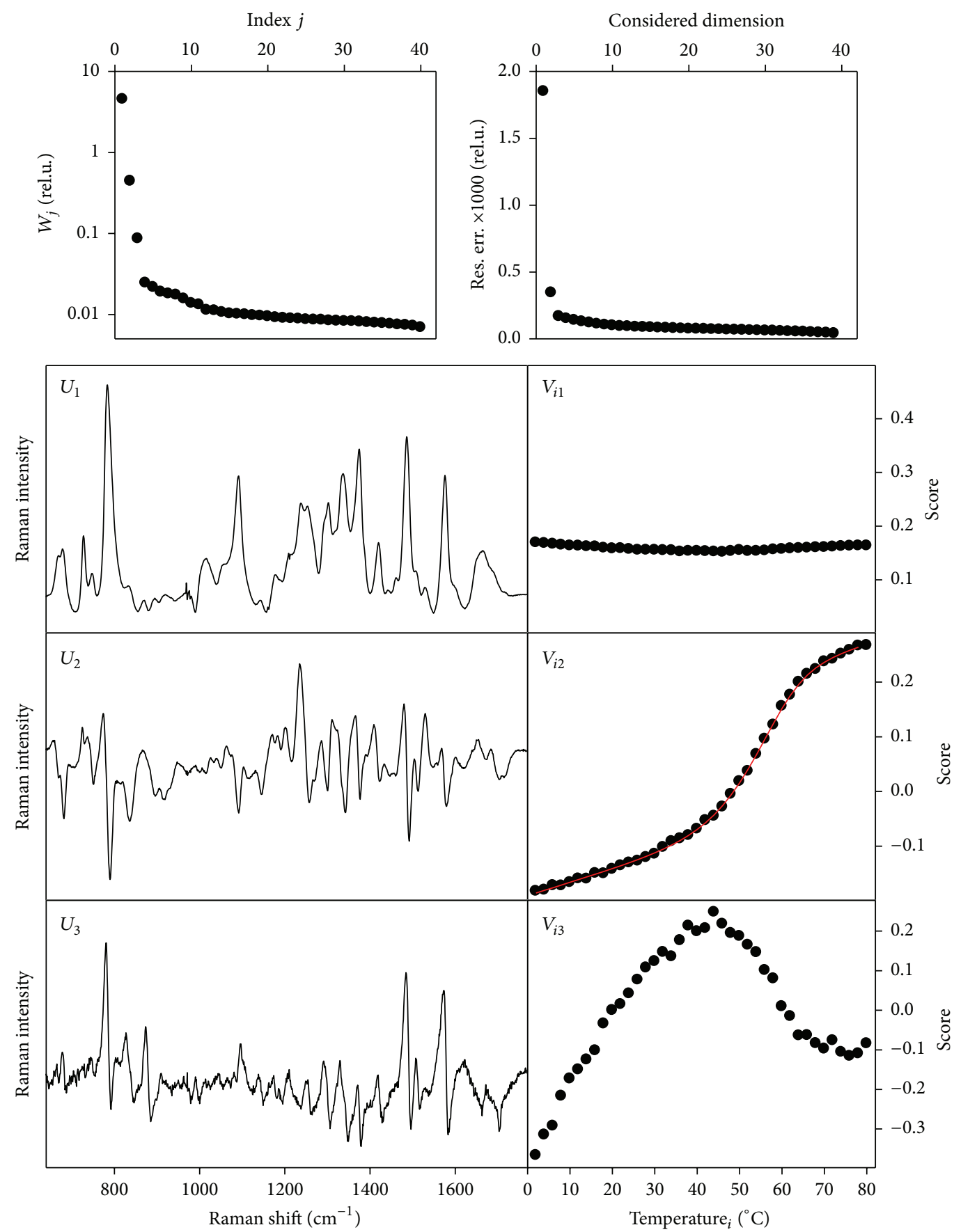

Figure 2: Typical results of singular values decomposition applied to the set of 40 temperature dependent Raman spectra of selfcomplementary DNA octamer (data for OctAT). The second spectral component $\left(U_{2}\right)$ reflects the main spectral changes connected with the duplex melting and the related scores $\left(V_{2}\right)$ were employed to determine the melting temperature by the least square fit. The result of the fit is shown in red.

origins of these two kinds of errors, the resulting value was calculated as a square root of a sum of squared partial errors.

Figure 4 shows the van't Hoff plot, that is, the graph of the reciprocal of the melting temperature in Kelvin plotted against the concentration in a logarithmic scale. If (4) is valid, the plot should be linear with its slope given by the enthalpy contribution. It can be directly seen that the cytosine methylation changes the slope for OctAT and OctTT, but in the opposite direction. On the other hand, the slope for OctAA is not changed.

Resulting $\Delta H$ and $\Delta S$ values were determined by nonlinear least square fits of data from Table 1 to (4). In each fit, the errors of $T_{m}$ were used as weights and the covariance matrix of $\Delta H$ and $\Delta S$ was estimated as the inverse of curvature near the minimum of chi-square calculated from the Jacobian. Considered errors of the concentration values 
TABLE 1: The melting temperatures determined by particular spectroscopic techniques.

\begin{tabular}{lcccccc}
\hline \multirow{2}{*}{ Sample } & \multicolumn{2}{c}{ UV absorption } & \multicolumn{2}{c}{ Raman scattering } \\
& $c(\mu \mathrm{M})$ & $T_{m}\left({ }^{1} \mathrm{C}\right)$ & $c(\mathrm{mM})$ & $T_{m}\left({ }^{\circ} \mathrm{C}\right)$ & $c(\mathrm{mM})$ & $T_{m}\left({ }^{\circ} \mathrm{C}\right)$ \\
\hline OctAT & $11.7 \pm 0.2$ & $36.4 \pm 0.5$ & $1.17 \pm 0.02$ & $50.7 \pm 0.6$ & $2.7 \pm 0.1$ & $54.6 \pm 0.8$ \\
mOctAT & $9.6 \pm 0.2$ & $38.2 \pm 0.6$ & $1.12 \pm 0.02$ & $52.4 \pm 0.5$ & $2.2 \pm 0.1$ & $54.4 \pm 0.9$ \\
OctTT & $12.0 \pm 0.2$ & $26.9 \pm 0.6$ & $1.06 \pm 0.02$ & $45.1 \pm 1.0$ & $2.8 \pm 0.1$ & $49.8 \pm 0.8$ \\
mOctTT & $12.6 \pm 0.2$ & $27.1 \pm 0.6$ & $0.92 \pm 0.02$ & $45.7 \pm 1.0$ & $2.9 \pm 0.1$ & $52.6 \pm 0.9$ \\
OctAA & $9.8 \pm 0.2$ & $35.6 \pm 0.5$ & $1.10 \pm 0.02$ & $51.0 \pm 0.6$ & $2.3 \pm 0.1$ & $53.3 \pm 1.0$ \\
mOctAA & $11.8 \pm 0.2$ & $37.7 \pm 0.6$ & $1.01 \pm 0.02$ & $51.9 \pm 0.6$ & $2.7 \pm 0.1$ & $57.0 \pm 1.2$ \\
\hline
\end{tabular}

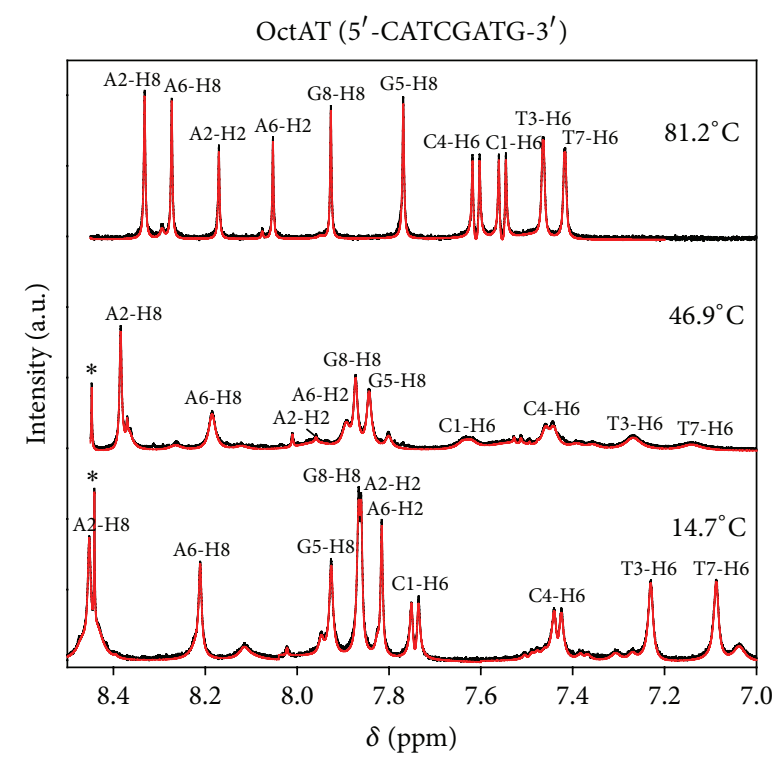

FIgURE 3: Example ${ }^{1} \mathrm{H}$ NMR spectra of OctAT in the region of nonexchangeable base hydrogen signals at temperatures when duplexes are formed (bottom), in the melting region (middle), and when only separated strands are present (top). Black lines show the experimental data and red ones the results of the fits (details in the text). The assignments of the NMR lines used for the melting temperature determination are denoted as the following: the type of the nucleobase, its position in the sequence from the $5^{\prime}$ side, and position of the hydrogen in respect of the purine or pyrimidine ring.

(Table 1), including those of the samples used in Raman measurements, were found to have only little influence on the final values of the thermodynamic quantities. We are aware of the low number of experimental data points in the van't Hoff fits. Nevertheless, this is overweighed by the fact that a quite wide concentration window is covered and that the individual melting temperatures are determined from careful analysis of large datasets. This makes our approach more reliable in obtaining $\Delta H$ and $\Delta S$ values than fitting melting curves for a narrow interval of concentrations.

Evaluated thermodynamic parameters $(\Delta H$ and $\Delta S$ and $\Delta G_{37^{\circ} \mathrm{C}}$ ) and their differences caused by the cytosine methylation $\left(\Delta \Delta H, \Delta \Delta S\right.$, and $\left.\Delta \Delta G_{37^{\circ} \mathrm{C}}\right)$ are shown in Table 2. Errors of the latter were estimated from assumption of independent error sources for particular samples. In fact, possible systematic shifts of the temperature scales for different

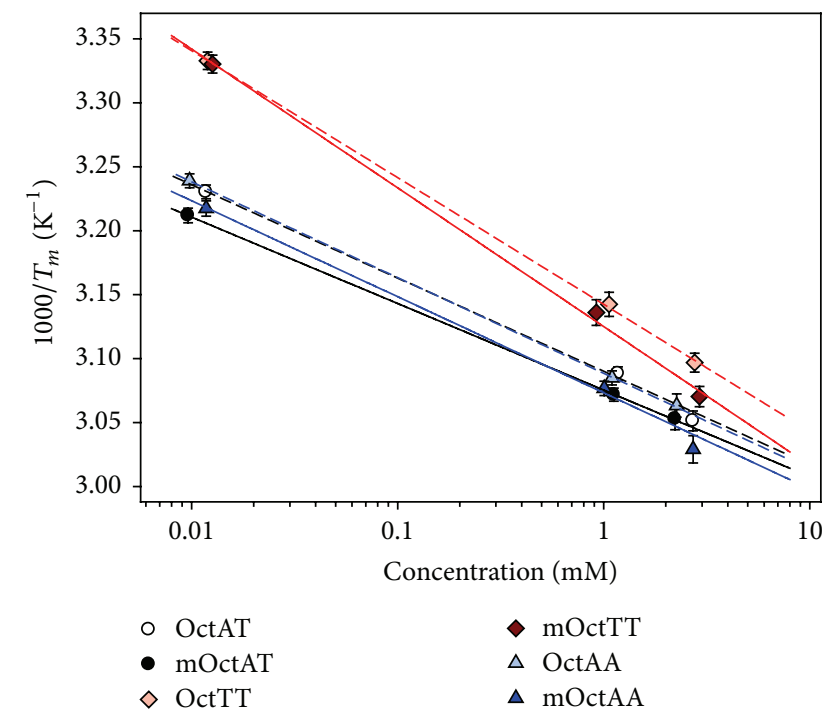

FIGURE 4: Van't Hoff plot of the melting temperatures determined for various octamers concentrations (see Table 1). The lines represent the best least square fits according to (4).

experimental equipment, which should also be considered, are not independent for different samples, but their effect is the same for both the nonmethylated and the methylated octamers and therefore does not contribute to $\Delta \Delta H, \Delta \Delta S$, and $\Delta \Delta G_{37^{\circ} \mathrm{C}}$ errors.

Despite the wide concentration window of almost three orders of magnitude and our care to determine correctly the melting temperature, the reliably estimated errors of $\Delta H$ and $\Delta S$ are relatively large in comparison with the changes caused by the methylation. Nevertheless, the observed changes lie outside the error intervals (except for OctAA) and clearly demonstrate the different thermodynamic signatures of the cytosine methylation for particular sequences (see Figure 4). The most obvious is the decrease of $\Delta H$ declination for OctTT, that is, for the sequence with TT..AA flanking nucleotides. Less pronounced (with respect to the estimated errors) is the opposite $\Delta H$ change for OctAT, that is, for AT..AT flanking nucleotides. In the case of OctAA (AA..TT) the thermodynamic effect of the methylation is not detectable. $\Delta H$ changes are accompanied with simultaneous changes of $\Delta S$, which might correlate with the enthalpyentropy compensation phenomenon [23, 24]. Anyway, our results demonstrate that it is the superposition of $\Delta H$ and 
TABLE 2: Thermodynamic characteristics of duplex formation and effect of the cytosine methylation.

\begin{tabular}{|c|c|c|c|c|c|c|}
\hline \multirow{2}{*}{$\begin{array}{l}\text { Sequence } \\
\text { Sample }\end{array}$} & \multicolumn{2}{|c|}{ CAT(m)CGATG } & \multicolumn{2}{|c|}{ CTT(m)CGAAG } & \multicolumn{2}{|c|}{ CAA(m)CGTTG } \\
\hline & OctAT & mOctAT & OctTT & mOctTT & OctAA & mOctAA \\
\hline$\Delta H\left(\mathrm{~kJ} \cdot \mathrm{mol}^{-1}\right)$ & $-262 \pm 12$ & $-283 \pm 14$ & $-193 \pm 8$ & $-176 \pm 7$ & $-255 \pm 12$ & $-255 \pm 13$ \\
\hline$\Delta \Delta H\left(\mathrm{~kJ} \cdot \mathrm{mol}^{-1}\right)$ & \multicolumn{2}{|c|}{$-21 \pm 18$} & \multicolumn{2}{|c|}{$17 \pm 11$} & \multicolumn{2}{|c|}{$0 \pm 17$} \\
\hline$\Delta S\left(\mathrm{~J} \cdot \mathrm{mol}^{-1} \cdot \mathrm{K}^{-1}\right)$ & $-753 \pm 37$ & $-813 \pm 44$ & $-549 \pm 26$ & $-494 \pm 23$ & $-731 \pm 36$ & $-726 \pm 41$ \\
\hline$\Delta \Delta S\left(\mathrm{~J} \cdot \mathrm{mol}^{-1} \cdot \mathrm{K}^{-1}\right)$ & \multicolumn{2}{|c|}{$-60 \pm 58$} & \multicolumn{2}{|c|}{$55 \pm 35$} & \multicolumn{2}{|c|}{$5 \pm 55$} \\
\hline$\Delta G_{37^{\circ} \mathrm{C}}\left(\mathrm{kJ} \cdot \mathrm{mol}^{-1}\right)$ & $-28.7 \pm 0.4$ & $-30.9 \pm 0.5$ & $-22.7 \pm 0.3$ & $-23.2 \pm 0.3$ & $-28.6 \pm 0.4$ & $-29.7 \pm 0.5$ \\
\hline$\Delta \Delta G_{37^{\circ} \mathrm{C}}\left(\mathrm{kJ} \cdot \mathrm{mol}^{-1}\right)$ & \multicolumn{2}{|c|}{$-2.1 \pm 0.7$} & \multicolumn{2}{|c|}{$-0.5 \pm 0.4$} & \multicolumn{2}{|c|}{$-1.2 \pm 0.6$} \\
\hline
\end{tabular}

$\Delta S$ contributions that causes the slight increase of the thermal stability after the methylation, observed for all studied sequences. The stabilization can also be seen in the decrease of $\Delta G$ values at $37^{\circ} \mathrm{C}$.

Among the three oligonucleotide sequences used in this work, the OctTT/mOctTT pair shows abnormally low stability while the other two sequences have their melting points and thermodynamic parameters similar to each other. This phenomenon was already observed for the nonmethylated OctTT by CD and NMR spectroscopy [35]. It was ascribed to an unusual BI to BII conformational exchange, accompanied with a structural kink and high twist and sugar pseudorotation phase angle at the CpG step in OctTT [17]. This is most probably caused by the TT/AA repeat as similar effect was also found in longer A-tracts [18]. Since this destabilization is not found in the OctAT sequence (which has the same central tetrad, TCGA), we conclude that the properties of $\mathrm{CpG}$ dinucleotide depend on more than just nearest neighboring residues.

The effect of the methylation on $\Delta H$ and $\Delta S$ values described in our work can hardly be compared with literature data, as the only published estimates are changes of $\Delta H$ obtained from NMR melting curves for a single sample concentration [21]. Although melting temperatures and changes of Gibbs free energies $\left(\Delta \Delta G_{37^{\circ} \mathrm{C}}=-1.6 \mathrm{~kJ} \cdot \mathrm{mol}^{-1}\right.$ for OctAT/mOctAT pair and $\Delta \Delta G_{37^{\circ} \mathrm{C}}=-0.5 \mathrm{~kJ} \cdot \mathrm{mol}^{-1}$ for OctTT/mOctTT pair) are determined precisely and are very close to the values we obtained in this work, $\Delta \Delta H$ values are highly inaccurate due to the above-mentioned $\Delta H$ and $\Delta S$ correlation.

\section{Conclusions}

We succeeded in obtaining reliable thermodynamic characteristics of DNA duplex formation for self-complementary octamers containing central CpG dinucleotide surrounded by a couple of AT pairs from both sides. The observed changes of the enthalpy and entropy contributions caused by the cytosine methylation exceed the estimated error intervals, which enables finding out strict dependence of this effect on the arrangement of the AT flanking nucleotide pairs. The enthalpy and entropy of duplex formation raise by the methylation in the case of the AT..AT surrounding, remain unchanged for the AA..TT surrounding, and even lower for the TT..AA sequence.
In general, our study demonstrates that combination of several spectroscopic techniques enables extension of the concentration window for reliable determination of enthalpy and entropy changes. However, our results also show that a care to minimize experimental errors is absolutely necessary and even then the precision of the determined values is limited.

\section{Conflict of Interests}

The authors declare that there is no conflict of interests regarding the publication of this paper.

\section{Acknowledgments}

The authors acknowledge Charles University (Project GAUK 430011) and the Czech Science Foundation (Project 1326526S) for financial support.

\section{References}

[1] C. Auclair, "Structural and functional regulation of DNA: geometry, topology and methylation," in Nanoscience, P. Boisseau, P. Houdy, and M. Lahmani, Eds., pp. 3-27, Springer, Berlin, Germany, 2009.

[2] H. Cedar and Y. Bergman, "Programming of DNA methylation patterns," Annual Review of Biochemistry, vol. 81, pp. 97-117, 2012.

[3] M. J. Ziller, H. Gu, F. Müller et al., "Charting a dynamic DNA methylation landscape of the human genome," Nature, vol. 500, no. 7463, pp. 477-481, 2013.

[4] A. M. Krieg, A.-K. Yi, S. Matson et al., "CpG motifs in bacterial DNA trigger direct B-cell activation," Nature, vol. 374, no. 6522, pp. 546-549, 1995.

[5] H. Hemmi, O. Takeuchi, T. Kawai et al., "A Toll-like receptor recognizes bacterial DNA," Nature, vol. 408, no. 6813, pp. 740$745,2000$.

[6] S. Morecki and S. Slavin, "Immunoregulation of GVHD by triggering the innate immune system with CpG," Expert Review of Hematology, vol. 2, no. 4, pp. 443-453, 2009.

[7] C. Bode, G. Zhao, F. Steinhagen, T. Kinjo, and D. M. Klinman, "CpG DNA as a vaccine adjuvant," Expert Review of Vaccines, vol. 10, no. 4, pp. 499-511, 2011.

[8] C. M. R. López, B. G. Asenjo, A. J. Lloyd, and M. J. Wilkinson, "Direct detection and quantification of methylation in nucleic 
acid sequences using high-resolution melting analysis," Analytical Chemistry, vol. 82, no. 21, pp. 9100-9108, 2010.

[9] M. Wanunu, D. Cohen-Karni, R. R. Johnson et al., "Discrimination of methylcytosine from hydroxymethylcytosine in DNA molecules," Journal of the American Chemical Society, vol. 133, no. 3, pp. 486-492, 2011.

[10] C. M. Rodríguez López, A. J. Lloyd, K. Leonard, and M. J. Wilkinson, "Differential effect of three base modifications on DNA thermostability revealed by high resolution melting," Analytical Chemistry, vol. 84, no. 17, pp. 7336-7342, 2012.

[11] D. Renciuk, O. Blacque, M. Vorlickova, and B. Spingler, "Crystal structures of B-DNA dodecamer containing the epigenetic modifications 5-hydroxymethylcytosine or 5-methylcytosine," Nucleic Acids Research, vol. 41, no. 21, pp. 9891-9900, 2013.

[12] J. A. Theruvathu, Y. W. Yin, B. M. Pettitt, and L. C. Sowers, "Comparison of the structural and dynamic effects of 5-methylcytosine and 5-chlorocytosine in a CpG dinucleotide sequence," Biochemistry, vol. 52, no. 47, pp. 8590-8598, 2013.

[13] K. B. Geahigan, G. A. Meints, M. E. Hatcher, J. Orban, and G. P. Drobny, "The dynamic impact of CpG methylation in DNA," Biochemistry, vol. 39, no. 16, pp. 4939-4946, 2000.

[14] S. Derreumaux, M. Chaoui, G. Tevanian, and S. Fermandjian, "Impact of CpG methylation on structure, dynamics and solvation of cAMP DNA responsive element," Nucleic Acids Research, vol. 29, no. 11, pp. 2314-2326, 2001.

[15] A. Thalhammer, A. S. Hansen, A. H. El-Sagheer, T. Brown, and C. J. Schofield, "Hydroxylation of methylated CpG dinucleotides reverses stabilisation of DNA duplexes by cytosine 5-methylation," Chemical Communications, vol. 47, no. 18, pp. 5325-5327, 2011.

[16] S. El Antri, O. Mauffret, M. Monnot, E. Lescot, O. Convert, and S. Fermandjian, "Structural deviations at $\mathrm{CpG}$ provide a plausible explanation for the high frequency of mutation at this site: phosphorus nuclear magnetic resonance and circular dichroism studies," Journal of Molecular Biology, vol. 230, no. 2, pp. 373-378, 1993.

[17] A. Lefebvre, O. Mauffret, E. Lescot, B. Hartmann, and S. Fermandjian, "Solution structure of the CpG containing d(CTTCGAAG) ${ }_{2}$ oligonucleotide: NMR data and energy calculations are compatible with a BI/BII equilibrium at CpG," Biochemistry, vol. 35, no. 38, pp. 12560-12569, 1996.

[18] C. Cordier, L. Marcourt, M. Petitjean, and G. Dodin, “Conformational variation of the central CG site in d(ATGACGTCAT) ${ }_{2}$ and d(GAAAACGTTTTC) $)_{2}$. An NMR, molecular modelling and 3D-homology investigation," European Journal of Biochemistry, vol. 261, no. 3, pp. 722-733, 1999.

[19] P. D. Dans, A. Pérez, I. Faustino, R. Lavery, and M. Orozco, "Exploring polymorphisms in B-DNA helical conformations," Nucleic Acids Research, vol. 40, no. 21, pp. 10668-10678, 2012.

[20] M. Pasi, J. H. Maddocks, D. Beveridge et al., " $\mu \mathrm{ABC}$ : a systematic microsecond molecular dynamics study of tetranucleotide sequence effects in B-DNA," Nucleic Acids Research, vol. 42, no. 19, pp. 12272-12283, 2014.

[21] A. Lefebvre, O. Mauffret, S. El Antri, M. Monnot, E. Lescot, and S. Fermandjian, "Sequence dependent effects of CpG cytosine methylation. A joint ${ }^{1} \mathrm{H}-\mathrm{NMR}$ and ${ }^{31} \mathrm{P}-\mathrm{NMR}$ study," European Journal of Biochemistry, vol. 229, no. 2, pp. 445-454, 1995.

[22] L. Marcourt, C. Cordier, T. Couesnon, and G. Dodin, "Impact of C5-cytosine methylation on the solution structure of d(GAAAACGTTTTC) ${ }_{2}$ : an NMR and molecular modelling investigation," European Journal of Biochemistry, vol. 265, no. 3, pp. 1032-1042, 1999.
[23] R. Baron and J. A. McCammon, "Molecular recognition and ligand association," Annual Review of Physical Chemistry, vol. 64, pp. 151-175, 2013.

[24] J. D. Chodera and D. L. Mobley, "Entropy-enthalpy compensation: role and ramifications in biomolecular ligand recognition and design," Annual Review of Biophysics, vol. 42, no. 1, pp. 121142, 2013.

[25] K. Wüthrich, "NOE-observable ${ }^{1} \mathrm{H}^{-1} \mathrm{H}$ distances in nucleic acids," in NMR of Proteins and Nucleic Acids, pp. 203-219, John Wiley \& Sons, Chichester, UK, 1986.

[26] J. Feigon, V. Sklenáŕ, E. Wang, D. E. Gilbert, R. F. Macaya, and P. Schultze, " $1 \mathrm{H}$ NMR spectroscopy of DNA," Methods in Enzymology, vol. 211, pp. 235-253, 1992.

[27] D. E. Wemmer, "Nucleic acid structure and dynamics from NMR," in NMR Spectroscopy and Its Application to Biomedical Research, S. K. Sarkar, Ed., pp. 281-312, Elsevier Science, Amsterdam, The Netherlands, 1996.

[28] A. V. Tataurov, Y. You, and R. Owczarzy, "Predicting ultraviolet spectrum of single stranded and double stranded deoxyribonucleic acids," Biophysical Chemistry, vol. 133, no. 1-3, pp. 66-70, 2008.

[29] H. Günther, "Experimental aspects of nuclear magnetic resonance spectroscopy," in NMR Spectroscopy. Basic Principles, Concepts, and Applications in Chemistry, pp. 53-67, John Wiley \& Sons, Chichester, UK, 2nd edition, 1995.

[30] J.-L. Mergny and L. Lacroix, "Analysis of thermal melting curves," Oligonucleotides, vol. 13, no. 6, pp. 515-537, 2003.

[31] R. Owczarzy, "Melting temperatures of nucleic acids: discrepancies in analysis," Biophysical Chemistry, vol. 117, no. 3, pp. 207215, 2005.

[32] V. Ř́mal, H. Štěpánková, and J. Štěpánek, "Analysis of NMR spectra in case of temperature-dependent chemical exchange between two unequally populated sites," Concepts in Magnetic Resonance Part A, vol. 38, no. 3, pp. 117-127, 2011.

[33] E. R. Malinowski, Factor Analysis in Chemistry, John Wiley \& Sons, 2002.

[34] W. H. Press, S. A. Teukolsky, W. T. Vetterling, and B. P. Flannery, "Modeling of data," in Numerical Recipes in C: The Art of Scientific Computing, pp. 656-706, Cambridge University Press, Cambridge, UK, 2nd edition, 1992.

[35] S. El Antri, P. Bittoun, O. Mauffret et al., "Effect of distortions in the phosphate backbone conformation of six related octanucleotide duplexes on CD and ${ }^{31} \mathrm{P}$ NMR spectra," Biochemistry, vol. 32, no. 28 , pp. 7079-7088, 1993. 

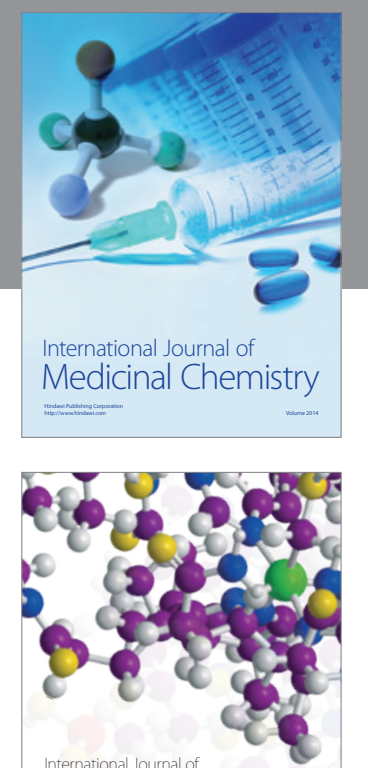

\section{Carbohydrate} Chemistry

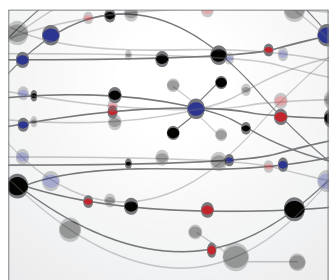

The Scientific World Journal
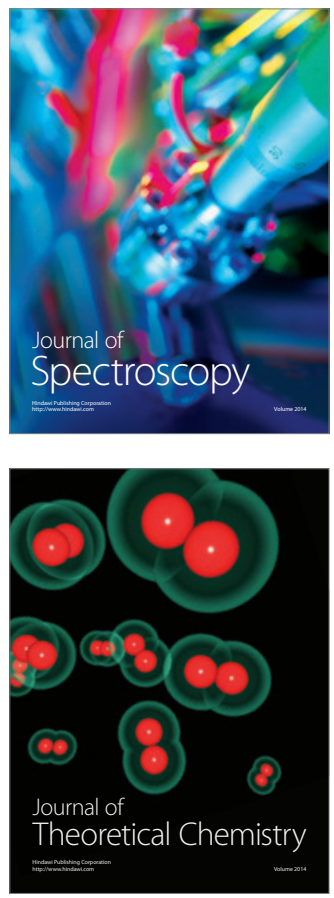
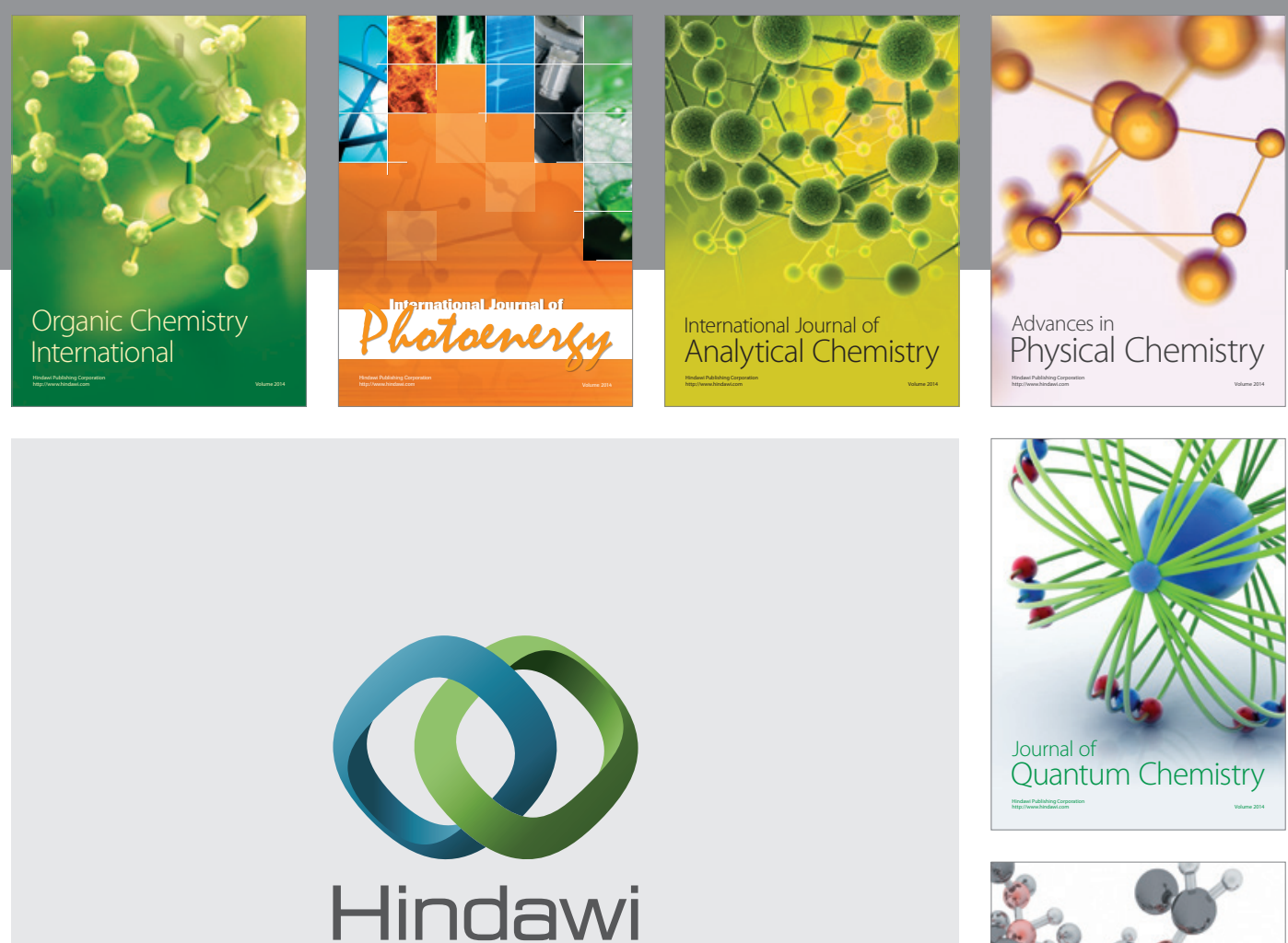

Submit your manuscripts at

http://www.hindawi.com

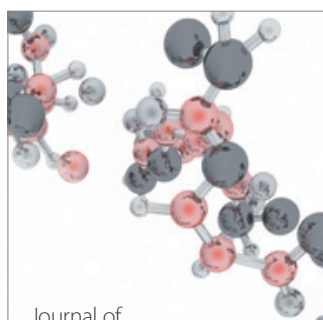

Analytical Methods

in Chemistry

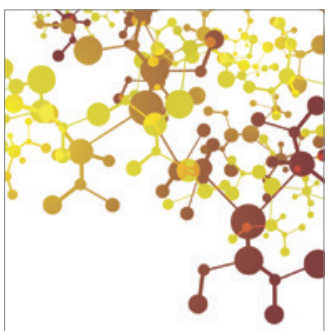

Journal of

Applied Chemistry

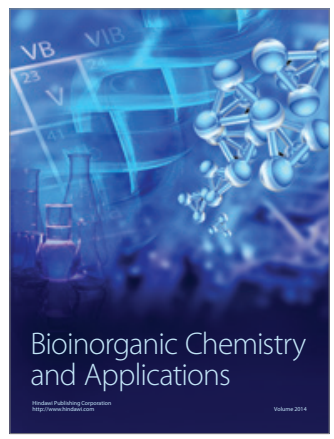

Inorganic Chemistry
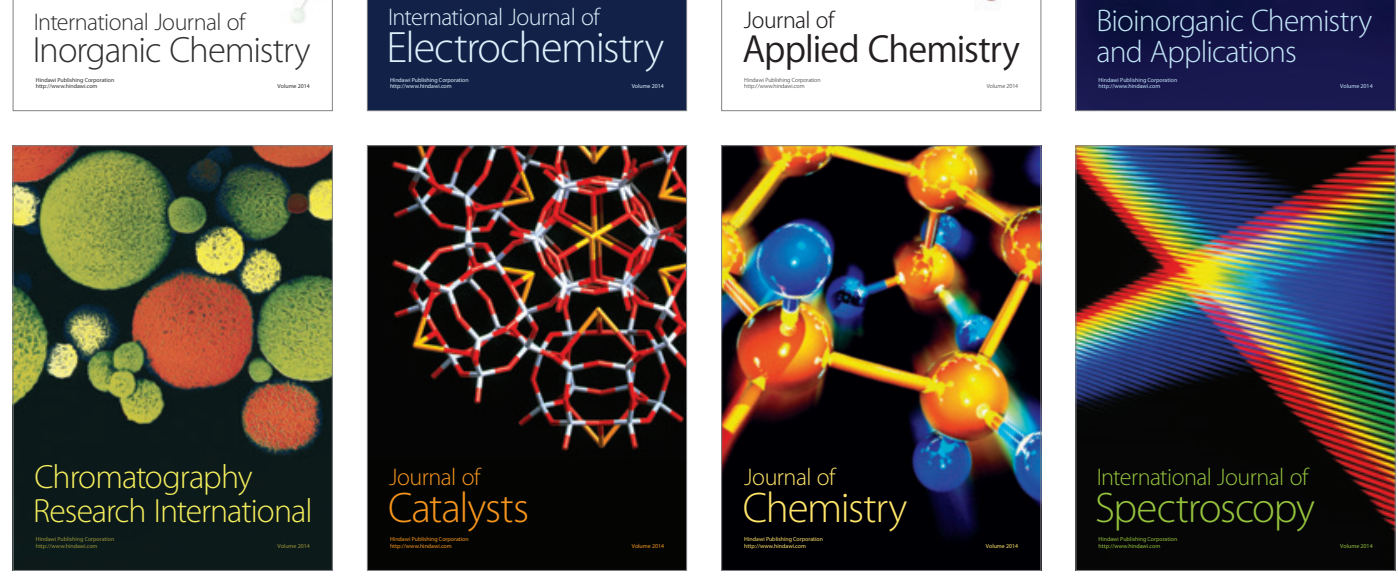